\title{
Enhanced protein adsorption and cellular adhesion using transparent titanate nanotube thin films made by a simple and inexpensive room temperature process: Application to optical biochips
}

\author{
Judit Nador ${ }^{\mathrm{a}, \mathrm{b}}$, Norbert Orgovan ${ }^{\mathrm{a}}$, Miklos Fried ${ }^{\mathrm{a}}$, Peter Petrik ${ }^{\mathrm{a}}$, Attila Sulyok ${ }^{\mathrm{a}}$, \\ Jeremy J. Ramsden ${ }^{\mathrm{c}}$, Laszlo Korosi ${ }^{\mathrm{d}}$, Robert Horvath ${ }^{\mathrm{a}, *}$ \\ a Nanobiosensorics Laboratory, Institute of Technical Physics and Materials Science, Research Centre for Natural Sciences, Hungarian Academy of Sciences, \\ Konkoly Thege M. út 29-33, Budapest H-1121, Hungary \\ ${ }^{\mathrm{b}}$ Doctoral School of Molecular- and Nanotechnologies, University of Pannonia, Egyetem u. 10., Veszprém H-8200, Hungary

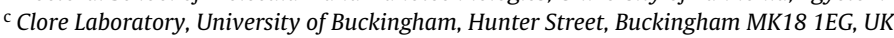 \\ d Department of Biotechnology, Nanophage Therapy Center, Enviroinvest Corporation, Kertváros u. 2., Pécs H-7632, Hungary
}

\section{A R T I C L E I N F O}

\section{Article history:}

Received 13 February 2014

Received in revised form 24 June 2014

Accepted 12 July 2014

Available online $\mathrm{xxx}$

\section{Keywords:}

Optical biosensor

Additive-free

Implant

Osteoblast

Cell adhesion

Nanostructured layer

\begin{abstract}
A B S T R A C T
A new type of titanate nanotube (TNT) coating is investigated for exploitation in biosensor applications. The TNT layers were prepared from stable but additive-free sols without applying any binding compounds. The simple, fast spin-coating process was carried out at room temperature, and resulted in well-formed films around $10 \mathrm{~nm}$ thick. The films are highly transparent as expected from their nanostructure and may, therefore, be useful as coatings for surface-sensitive optical biosensors to enhance the specific surface area. In addition, these novel coatings could be applied to medical implant surfaces to control cellular adhesion. Their morphology and structure was characterized by spectroscopic ellipsometry (SE) and atomic force microscopy (AFM), and their chemical state by X-ray photoelectron spectroscopy (XPS). For quantitative surface adhesion studies, the films were prepared on optical waveguides. The coated waveguides were shown to still guide light; thus, their sensing capability remains. Protein adsorption and cell adhesion studies on the titanate nanotube films and on smooth control surfaces revealed that the nanostructured titanate enhanced the adsorption of albumin; furthermore, the coatings considerably enhanced the adhesion of living mammalian cells (human embryonic kidney and preosteoblast).
\end{abstract}

(c) 2014 Elsevier B.V. All rights reserved.

\section{Introduction}

The sensing of biomacromolecule concentration, affinity or adsorption using modern, label-free methods such as OWLS and QCM [1], requires that the objects to be sensed (e.g., proteins) are first captured by a sensor. Hence, it is advantageous for the sensor surface to be as large as possible because more analytes can be captured. Although many techniques for surface structuring of materials exist [2], a material that can simply be applied as a coating to practically any surface is attractive. In this paper, we propose the use of the recently created titanate nanotubes as such a coating.

Protein adsorption on surfaces is important in many fields of bio- and nanotechnology, e.g., for biosensors and biochips, drug delivery materials [3-5], implant and other medical device coatings

\footnotetext{
* Corresponding author. Tel.: +36 705490732 .

E-mail addresses: horvathr@mfa.kfki.hu, r74horvath@gmail.com (R. Horvath).
}

[6], or the fabrication of novel hybrid materials [7]. The biosensor or biochip typically uses enzymes or antibodies immobilized on the sensing surfaces to create a functionalized capture layer [8-11]. The development of biocompatible materials [12-15] may also involve coating them with proteins and peptides, which usually play an essential role in the control of cellular adhesion and has a direct influence on the behavior and other characteristics of living cells (the extent and strength of adhesion, whether a cell undergoes apoptosis, proliferation or differentiation) [16,17]. Phenomenologically the attachment and spreading of cells are regulated by various biophysicochemical properties of their substrata, particularly biochemical composition, topography, roughness and mechanical properties [18,19].

Although in certain cases, the reduction or even, if achievable, the prevention of protein and cellular adhesion is desirable, e.g., for antifouling surfaces and medical devices implanted into the bloodstream [13,20-24], in most cases, such as medical implants designed to become assimilated with their host tissue, the 
enhancement of surface adhesion is advantageous [2,13,25]. A commonly used strategy to improve adhesion is to increase the specific area of the surface [26-28]. In surface-sensitive biosensing, increasing the specific area of the sensing surface improves the sensitivity, because more target analyte can be captured. One possible way to enlarge the specific area is via surface nanostructuring [29-32]. However, in the optical techniques an important aspect is that the modification of the sensor must not quench the propagation of light, hence scattering and absorption have to be minimized.

In the present work, we demonstrate that titanate nanotubes (TNT) are especially promising candidates for making additive-free, transparent nanostructured surfaces. These novel coatings are prepared from pure sols by spin-coating at room temperature without additional binding components.

Previous works demonstrated immobilized protein activity [33], enhanced protein adsorption [34] and cell behavior [35,36] on various nanostructured coatings.

Our titanate nanotubes have a structure similar to a rolledup carpet, and are up to $100-500 \mathrm{~nm}$ long [37], with an outer diameter of $7-11 \mathrm{~nm}$ and an inner diameter of 4.5-6.5 nm [38] (and can potentially be used for delivery by storing drugs or other biomolecules in their inner volume [39]). It has already been demonstrated that biodegradable polymer scaffolds can easily be coated with these films, hence opening applications in tissue engineering and regenerative medicine [40].

Waveguide sensors were recently exploited in several novel directions; such as the on-line monitoring of oriented protein adsorption [41], extracellular vesicles [42] and human primary immune [16] and cancer [17] cell adhesion in a completely labelfree manner. Here we report on the preparation of TNT coatings on surface-sensitive optical waveguide-based biosensors for quantitative adsorption and adhesion studies. The morphology of the layers was characterized by atomic force microscopy (AFM) and spectroscopic ellipsometry (SE). The chemical states of the coatings and control surfaces were investigated using X-ray photoelectron spectroscopy (XPS). We demonstrate that these films considerably enhance the adsorption of proteins and the adhesion of living cells, opening the way for further applications in optical biosensorics.

\section{Materials and methods}

\subsection{TNT sol preparation}

The TNT sol used in the coating process was prepared using a previously reported protocol [37].

\subsection{Substrate cleaning and coating procedures}

Prior to spin-coating, the optical chips were steeped in chromic acid (Merck) for $3 \mathrm{~min}$, then in milli-Q water (MQ) for $2 \mathrm{~s}$, in $0.5 \mathrm{M}$ $\mathrm{KOH}$ solution (Merck, analytical grade) for $10 \mathrm{~s}$ and in water again. Finally, the chips were sonicated with an S $15 \mathrm{H}$ Elma sonicator for about $30 \mathrm{~min}$, changing the MQ water over them 10 times during sonication.

The Si plates were cleaned with hot 'Piranha solution' (a 3:1 mixture of $95 \% \mathrm{H}_{2} \mathrm{SO}_{4}$ (AnalaR NORMAPUR ${ }^{\circledR}$ ACS) and $30 \% \mathrm{H}_{2} \mathrm{O}_{2}$ (Scharlab EMSURE ${ }^{\circledR}$ ACS)) for 15 min and subsequently copiously rinsed with MQ water. The glass plates were sonicated in Cobas Integra cleaner solution $(300 \mathrm{mM} \mathrm{HCl}, 1 \%$ detergent, Roche Diagnostics) and in water for $15-15 \mathrm{~min}$ and then exposed to an $\mathrm{O}_{2}$ plasma (Plasma Prep II, Structure Probe Inc.) for 5 min.

The films were prepared from the TNT sol on OW2400 (Microvacuum Ltd.) $\mathrm{Si}_{0.6} \mathrm{Ti}_{0.4} \mathrm{O}_{2}$ optical chips, on $1 \mathrm{~cm} \times 1 \mathrm{~cm}$ pieces of silicon $(1,0,0)$ substrates (covered by native oxide $3 \mathrm{~nm}$ thick) and on microscope slides (MENZEL-GLÄSER, $1 \mathrm{~mm}$ thick, Thermo
Scientific). For coating, a Convac St 450 spin-coater was used at $3000 \mathrm{rpm}$, for $25 \mathrm{~s}$.

\subsection{TNT film characterization techniques}

The topography of the TNT layers was studied by AFM (Aist-NT, DigiScope1000) in tapping mode.

The thickness and millimeter-scale homogeneity of the TNT films on the silicon plates were characterized by SE (Woollam M2000DI) in mapping mode. The angles of incidence of the mapping beam were $70^{\circ}$ and $75^{\circ}$. The results were evaluated with CompleteEASE 4.72 software.

The chemical state of components at the surface was examined by X-ray photoelectron spectroscopy (XPS) using an Al anode. Spectra were obtained by a special cylindrical mirror analyser with retarding field (type ESA 105, Staib Instruments Ltd) that provided a constant energy resolution of $1.5 \mathrm{eV}$. Titanium $2 \mathrm{p} 3 / 2$ and oxygen $1 \mathrm{~s}$ lines were detected at binding energies of $454 \mathrm{eV}$ and $531 \mathrm{eV}$, respectively. After taking a spectrum from the freshly prepared surface, further spectra were recorded after applying low energy ion bombardment $\left(\mathrm{Ar}, 1 \mathrm{keV}, 78^{\circ}\right.$ angle of incidence) to remove possible surface contamination from the air environment. The subcomponents within the detected XPS lines were calculated according to a general evaluation procedure, including Shirley background subtraction and Gaussian/Lorentzian fitting. It resulted in the separation of the oxygen of $\mathrm{TiO}_{2}$ and of $\mathrm{OH}$ groups.

\subsection{Protein solutions and cell cultures}

$10 \mathrm{mM}$ phosphate buffer was prepared from phosphatebuffered saline (PBS) tablets (Sigma-Aldrich, P4417-100TAB) dissolved in MQ and sonicated for 10-15 min. $1 \mathrm{mM}$ bovine serum albumin (BSA) solution was made from powder (Sigma-Aldrich, A7906-10G) dissolved in $10 \mathrm{mM}$ PBS. Human embryonic kidney (HEK293) and preosteoblast (MC 3T3-E1) cells were cultured in an incubator $\left(37^{\circ} \mathrm{C}, 5 \% \mathrm{CO}_{2}, 20 \% \mathrm{O}_{2}\right)$ in Dulbecco's modified Eagle's medium (DMEM) and alpha-MEM respectively, both supplemented with $10 \%$ fetal bovine serum (FBS), $4 \mathrm{mM} \mathrm{L-glutamine,} 40 \mu \mathrm{g} \mathrm{mL}^{-1}$ gentamycin and $0.25 \mu \mathrm{g} \mathrm{mL} \mathrm{L}^{-1}$ amphotericin.

Both types of the cells were trypsinized with $1.0 \%$ trypsin-EDTA warmed to $37^{\circ} \mathrm{C}$. Trypsin was removed before the cells detached completely. The activity of the trypsin was arrested by adding culture medium containing 10\% FBS. Harvested cells were centrifuged at $380 \times \mathrm{g}$ for $7 \mathrm{~min}$ and the cell pellet was resuspended in assay buffer with intensive but gentle pipetting.

\subsection{Biosensing measurements and optical imaging}

Protein adsorption was measured using an OWLS210 integrated optical scanning instrument (Microvacuum Ltd, Hungary) with Biosense 3.0 and Biosense 2.8 software and cell adhesion [43-46] with a laboratory-built OWLS instrument and an ASI BIOS-1 instrument (Microvacuum Ltd, Hungary) based on the same working principle [47]. Both instruments used a wavelength of $623.82 \mathrm{~nm}$ for the illuminating beam. ${ }^{1}$ Each experiment was typically repeated three times.

\footnotetext{
1 Optical waveguide lightmode spectroscopy (OWLS) is based on the incoupling of a monochromatic (e.g., He-Ne laser) beam by a shallow surface grating into a high refractive index waveguide layer. The sensor chip is rotated by a high precision goniometer relative to the illuminating beam and the incoupling angles are determined from the positions of sharp resonant peaks in the coupled light intensity-illumination angle function. From the measured incoupling angles the adsorbed protein quantity can be calculated $[1,69]$.
} 

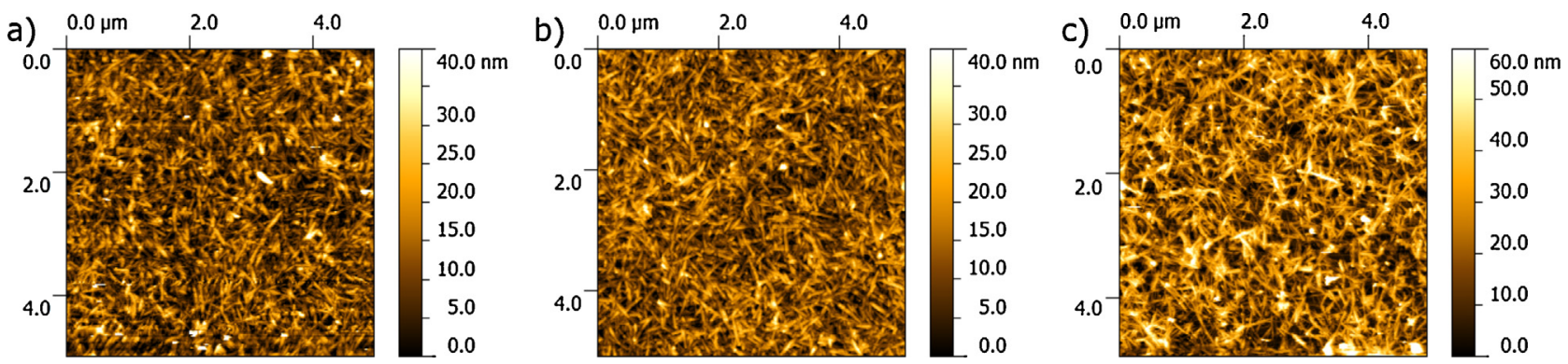

Fig. 1. Representative AFM images of the TNT layers coating a silicon wafer (a), an OWLS chip (b), and a glass microscope slide (c).

Images of surface-adhered cells were taken by an inverted phase contrast microscope (Zeiss Axiovert Observer) using $20 \times$ objectives.

\section{Results and discussions}

\subsection{Surface morphology and optical characterization of the titanate-nanotube (TNT) films}

The first aim was to cover an optical waveguide lightmode spectroscopy (OWLS) sensor chip (OW2400, Microvacuum Ltd., Hungary) with a TNT film that is compatible with OWLS measurements. Ideally the layer should be homogeneous, dense and with a thickness between 5 and $15 \mathrm{~nm}$, at which it can be expected to well cover the chip, but does not significantly increase the optical thickness of the original waveguiding layer (geometric thickness typically $170-180 \mathrm{~nm}$, with a refractive index of about 1.8) [48].

The TNT films were characterized by atomic force microscopy (AFM) [49-51], which revealed that the nanotubes well covered the whole surface of the silicon wafer, the OWLS chip and the glass plate homogeneously (Fig. 1) The arithmetic average roughness $\left(R_{\mathrm{a}}\right)$ and root mean squared roughness (RMS) [13,52] of the surface were calculated from the AFM data, and were approximately $3.9 \mathrm{~nm}$ and $5.3 \mathrm{~nm}$, respectively. The uncoated surfaces were featureless with $R_{\mathrm{a}}<1 \mathrm{~nm}$. We attempted to remove the nanotubes by scratching with the AFM tip, but they were too strongly adherent to be removed. From cross sections of the topography the coating thickness was estimated as $12 \pm 2 \mathrm{~nm}$.

The thicknesses of the TNT films prepared on small pieces of silicon wafers were characterized by spectroscopic ellipsometry (SE) [53-55]. In this technique, an optical model is created based on a priori information and reasonable assumptions about the sample structure. The parameters of the optical model were fitted in order to minimize the difference between the measured and the calculated spectra (Fig. 2). We used a nonlinear least squares gradient fitting algorithm to minimize the mean square error (MSE) defined by

$\operatorname{MSE}=1000\left\{(3 n-p)^{-1} \sum_{i=1}^{n}\left[\left(N_{M_{i}}-N_{C_{i}}\right)^{2}+\left(C_{M_{i}}-C_{C_{i}}\right)^{2}+\left(S_{M_{i}}-S_{C_{i}}\right)^{2}\right\}^{1 / 2}\right.$

where $N=\cos (2 \Psi), C=\sin (2 \Psi) \cos (\Delta), S=\sin (2 \Psi) \sin (\Delta)$, with $\Psi$ and $\Delta$ corresponding to the amplitude and phase change during reflection of polarized light from the sample surface, respectively. $n$ and $p$ denote the number of wavelengths and the number of fitted parameters, respectively. Subscripts $M$ and $C$ signify "Measured" and "Calculated" data, respectively, and are themselves subscripted with the wavelength index ( $i$ ). The repeatability for $\Psi$ and $\Delta$ are better than 0.05 in the whole wavelength range, which allows a subnanometer sensitivity for the layer thickness to be achieved.

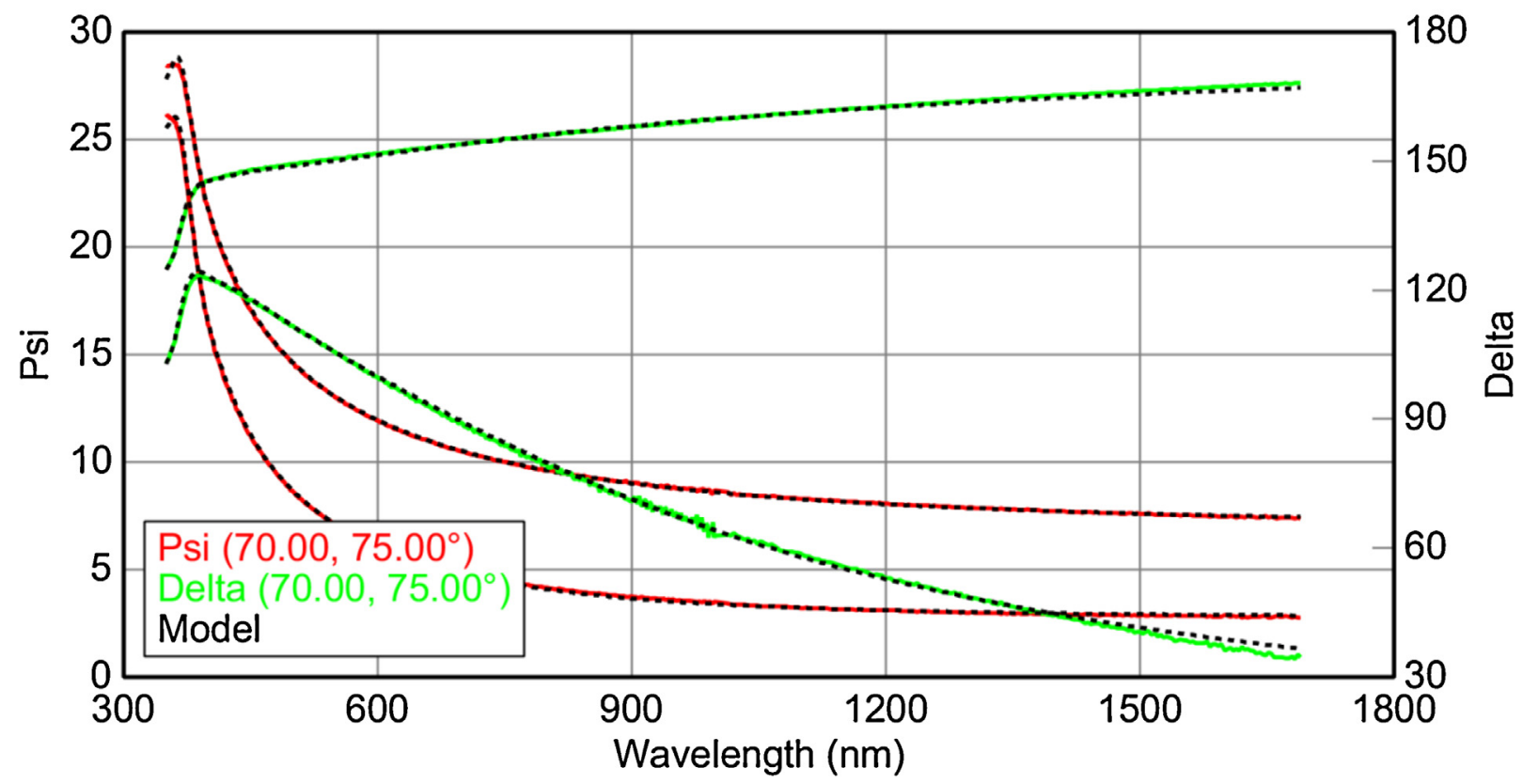

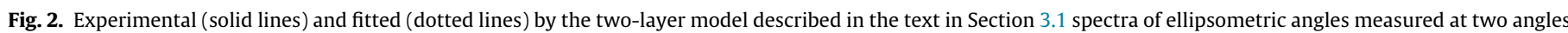
of incidence.

Please cite this article in press as: J. Nador, et al., Enhanced protein adsorption and cellular adhesion using transparent titanate nanotube thin films made by a simple and inexpensive room temperature process: Application to optical biochips, Colloids Surf. B: Biointerfaces (2014), http://dx.doi.org/10.1016/j.colsurfb.2014.07.015 
a)

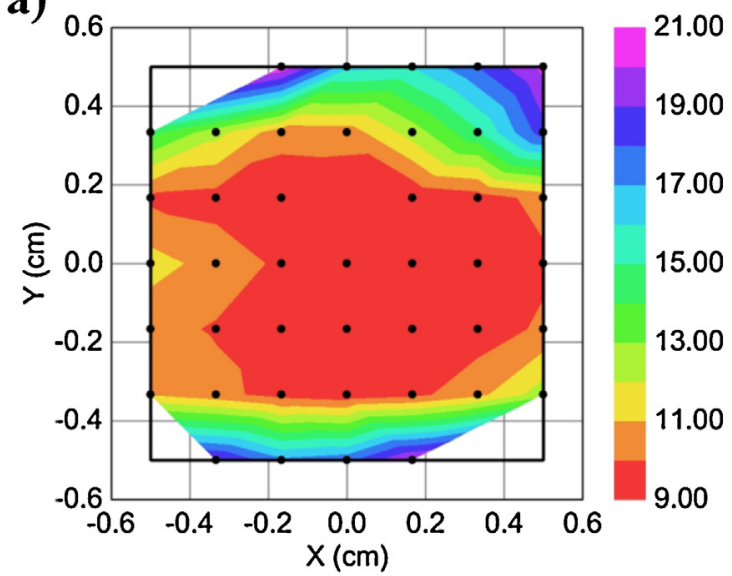

b)

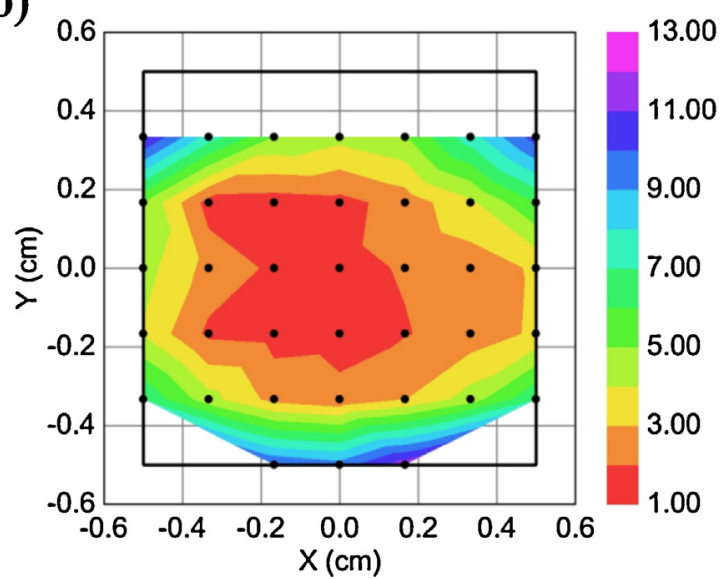

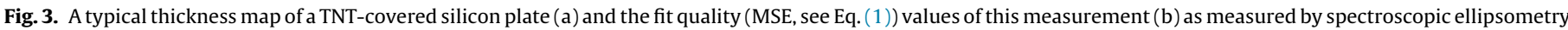

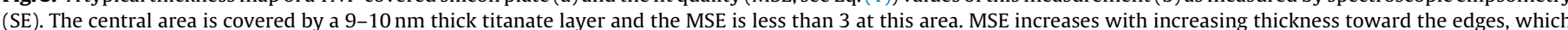
may indicate decreasing layer quality (possibly a result of the spin-coating), and inapplicability of the two-layer model.

MSE values close to 1 represent a high quality fit. For complex (multilayer) samples, MSE values below 10 are acceptable.

We used a two-layer optical model with the single-crystalline Si substrate: a native oxide layer (the thickness of which was fixed at the value obtained from measurements before the preparation of the TNT layers), and a TNT layer. The refractive index of the TNT layer was described by the Cauchy dispersion relation [56], an empirical polynomial approach for refractive index function of insulators and semiconductors below the band-gap [57]. The fit quality significantly improved when using a vertical grading of the refractive index within the Cauchy layer, incorporated in the CompleteEASE software used for the evaluation. Grading is implemented in the form of sublayers (in our case, 5). The refractive index changes from sublayer to sublayer as a predefined function of depth; in our case, a linear gradient was used. The inhomogeneity, which describes the change of the refractive index from the top to the bottom sublayer (a positive value corresponding to increasing refractive index toward the substrate), was typically found to be $60 \%$.

The mapping measurements revealed that the greatest part of the total area was covered with a 9-13 nm thick TNT layer. The most important area on the OWLS chip is the $1 \mathrm{~mm}^{2}$ sensing zone on the grating in the middle of the sensor chip. According to the SE maps in Fig. 3, the thickness of the TNT coverage is uniform within this crucial (for sensing purposes) central area.

\subsection{Light-guiding capabilities of the TNT-coated OWLS chips}

As mentioned, a coating is obviously useless for optical biosensing applications if it quenches light propagation. Fig. 4 shows the resonant incoupling peaks of typical uncoated and TNT-coated OWLS chips. The resonant peaks (from left to right, $\mathrm{TM}_{0}$ and $\mathrm{TE}_{0}$ ) are shifted to higher angles by the coating. The refractive index and the thickness of the guiding film were determined from the positions of the peaks before and after the coating procedure $[1,48,58]$. After coating the refractive index $\left(n_{\mathrm{F}}\right)$ decreased from 1.77494 to 1.77217 and the thickness $\left(d_{\mathrm{F}}\right)$ increased from $175.44 \mathrm{~nm}$ to $183.37 \mathrm{~nm}$, similar to the result of the ellipsometric measurements. ${ }^{2}$

2 According to the 3-layer model (i.e., the $\mathrm{Si}_{0.6} \mathrm{Ti}_{0.4} \mathrm{O}_{2}$ and TNT layers are approximated as a single uniform layer of an average refractive index).

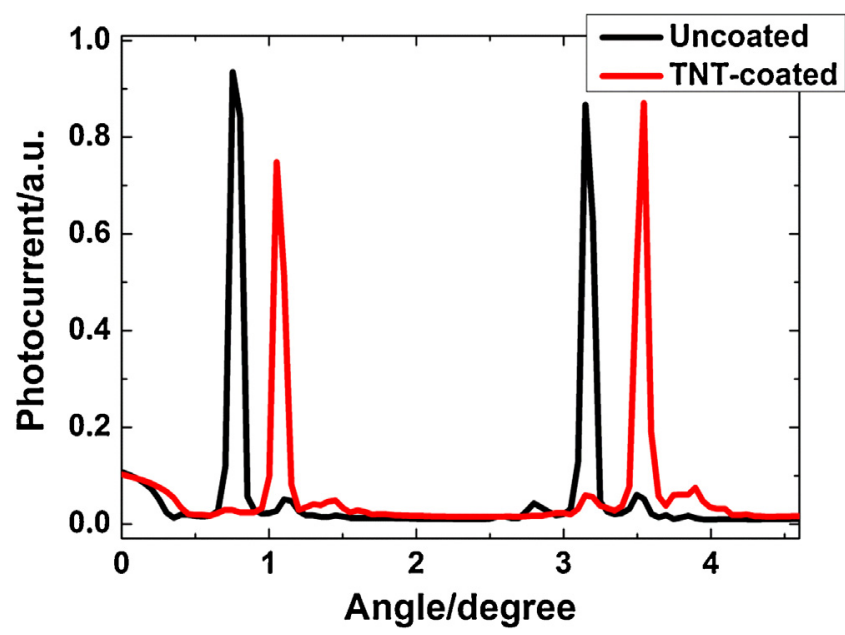

Fig. 4. The resonant incoupling peaks of a typical uncoated and a TNT-coated OWLS chip. The ordinate plots the current output from the photodiodes positioned at the ends of the waveguide.

\subsection{Protein adsorption experiments on the TNT-coated and uncoated OWLS chip}

First, a baseline was established with buffer flowing above the chip for 1-2h. Then the sample was introduced into the cuvette with a peristaltic REGLO Digital MS-4/12 (Ismatec) pump (flow rate $1 \mu \mathrm{Ls}^{-1}$ ). Adsorption was monitored for $2 \mathrm{~h}$, followed by a washing step during which pure buffer flowed at $1 \mu \mathrm{Ls}^{-1}$. The temperature at the OWLS cuvette was kept at $25^{\circ} \mathrm{C}$ during all experiments.

After washing, the surface density of the residual (after washing) adsorbed mass of a relatively small $(66.5 \mathrm{kDa})$ protein, BSA, was $130 \pm 20 \mathrm{ng} \mathrm{cm}^{-2}$ on the uncoated chip, and $250 \pm 20 \mathrm{ng} \mathrm{cm}^{-2}$ on the TNT-coated chip (Fig. 5). Adsorption on the uncoated $\mathrm{Si}_{0.6} \mathrm{Ti}_{0.4} \mathrm{O}_{2}$ chip resulted in an essentially stable monolayer as observed previously [59]. Adsorption was increased by $92 \pm 16 \%$ on the nanostructured titanate coating, while still resulting in an essentially stable monolayer (as inferred from the saturation of the adsorption with minor desorption after removal of the protein from the bulk solution).

Note that the factor that limits the protein desorption is the very high energy barrier due to the strong interfacial interaction. Unlike in the case of the cells (see below) even at flow rates of thousands of $\mu \mathrm{Ls}^{-1}$ the width of the diffusion boundary layer is 


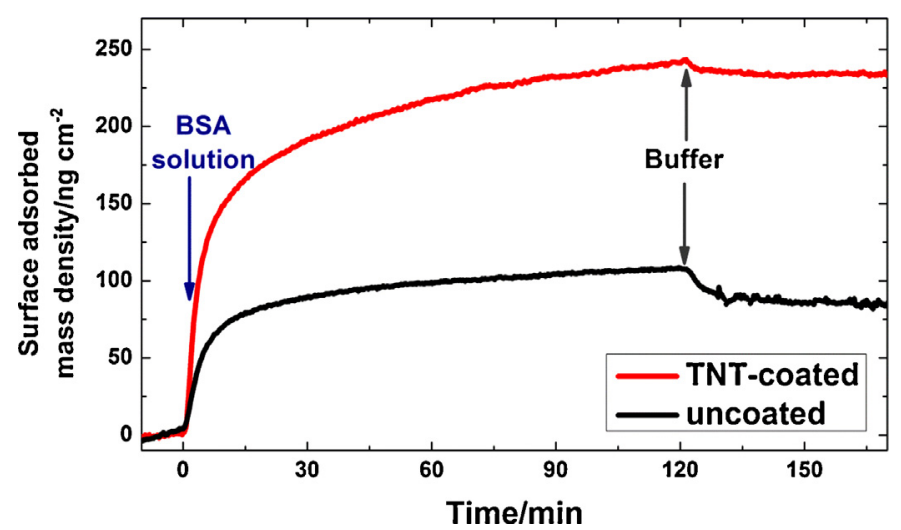

Fig. 5. Representative OWLS measurements of protein (BSA) adsorption on bare and TNT-coated chips. The ordinate plots the mass of adsorbed protein calculated from the raw OWLS data [1].

orders of magnitude greater than the size of the protein molecule and there is no hydromechanical force on the protein $[59,60]$.

\subsection{Cell spreading kinetics and adhesion strength on the TNT-coated and uncoated surfaces}

Measurements were made with HEK293 cells using the laboratory-built OWLS device dedicated to live cell studies, with the temperature at the OWLS chip being kept at $37^{\circ} \mathrm{C}$ throughout the experiments and a closed cuvette without flow placed over the sensing area enabling the cells to be introduced using a pipette [60]. First, the position of the incoupled resonant peaks were recorded when the cuvette contained buffered cell culture medium without cells. After establishing a stable baseline, $500 \mu \mathrm{L}$ of medium containing 25,000 cells were pipetted into the cuvette and the resonant peaks were measured for about 2 more hours. As the cells adhered and spread on the surface, the resonant peaks shifted to higher angles. A larger OWLS signal was obtained with the TNTcoated surface (Fig. 6), clearly indicating that the nanostructured titanate coating facilitates the adhesion of living cells [61].

In the next experiment, HEK293 cells were cultured on TNTcoated and uncoated glass plates in an incubator and phase contrast images were taken each day with an inverted microscope (Fig. 7). The microscope images of the two plates looked similar for six days. On the seventh day the cells were washed using an intense flow $(350 \mu \mathrm{L} / \mathrm{s})$ of cell culture medium and the surfaces were again observed under the microscope. While most of the cells were removed from the uncoated plates, the cell number on the

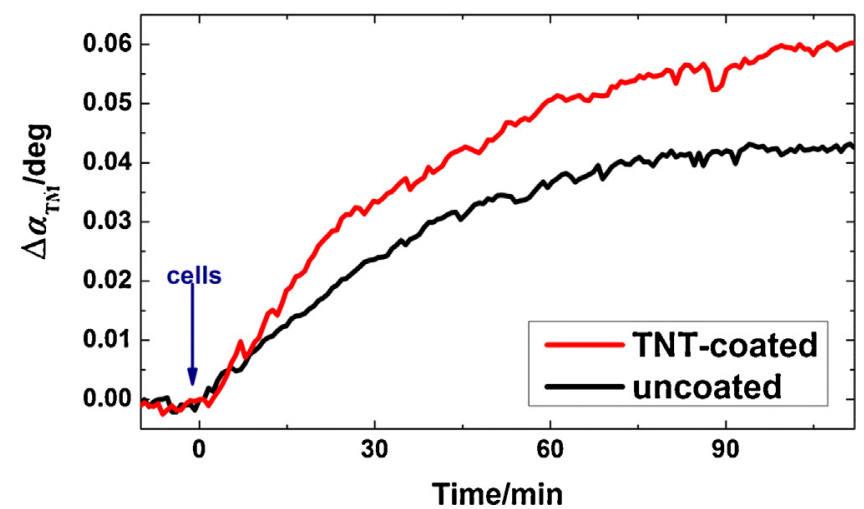

Fig. 6. OWLS measurement of HEK293 cell attachment and rapid spreading [68] on bare and TNT-covered sensor chips. The ordinate plots the shift of the peak position, in degrees, of the zeroth transverse magnetic guide lightmode, which is proportional to the cell contact area [61].
Uncoated plate

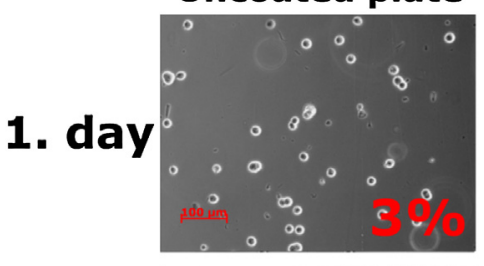

TNT-coated plate
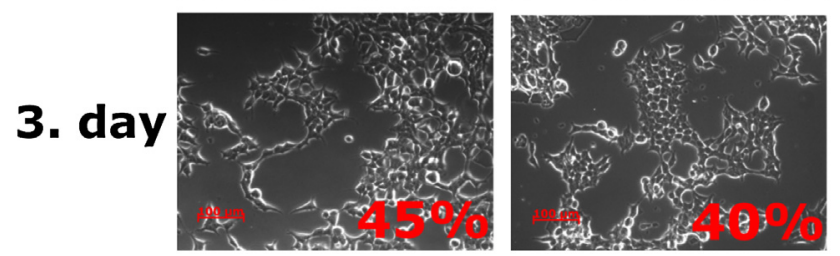

\section{6. day}
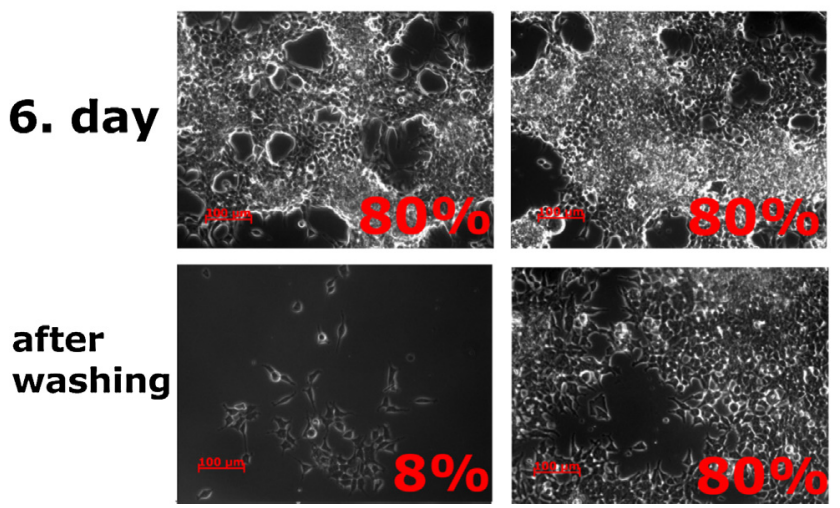

Fig. 7. Phase contrast images of HEK293 cells 1 day, 3 days and 6 days after seeding on uncoated and TNT-coated glass, and after washing with medium. On each image the estimated area covered by cells (calculated by using Image J software) are indicated.

TNT-coated surface remained almost the same as before washing. These experiments revealed that the cells adhered much more strongly on the TNT layer.

A more relevant cell type for possible biomaterials applications, a preosteoblast cell line, was also tested. Importantly, in this case the above-applied washing could not remove the cells, neither from the titanate-coated glass plates nor from the control glass plates, indicating a higher adherence for this cell type.

The adhesion kinetics of the preosteoblast cells were also investigated using OWLS, in a similar way as for the HEK cells (Fig. 8). The OWLS signals obtained were significantly larger than those from

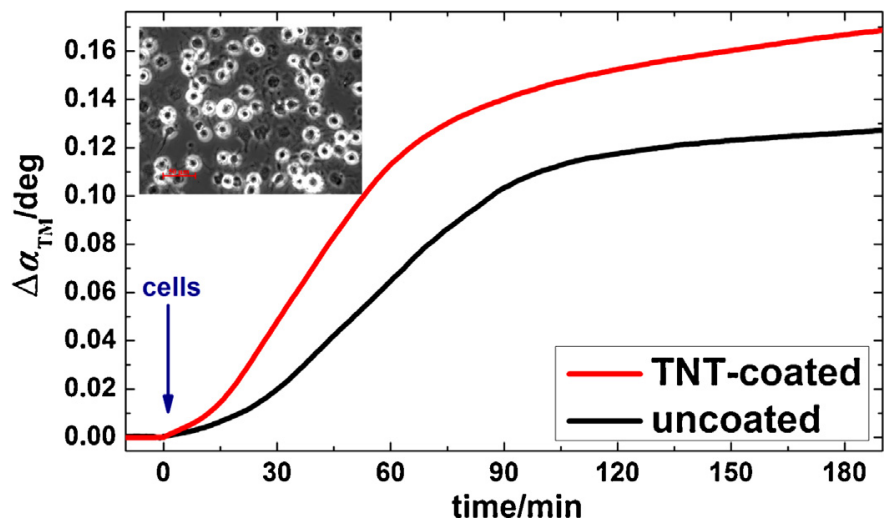

Fig. 8. OWLS measurement of preosteoblast cell (MC 3T3-E1) attachment and rapid spreading on bare and TNT-covered sensor chips. The ordinate plots the shift of the peak position, in degrees, of the zeroth transverse magnetic guide lightmode, which is proportional to the cell contact area. The insert shows the spread cells after the experiment (the scale bar is $50 \mu \mathrm{m}$ ). 

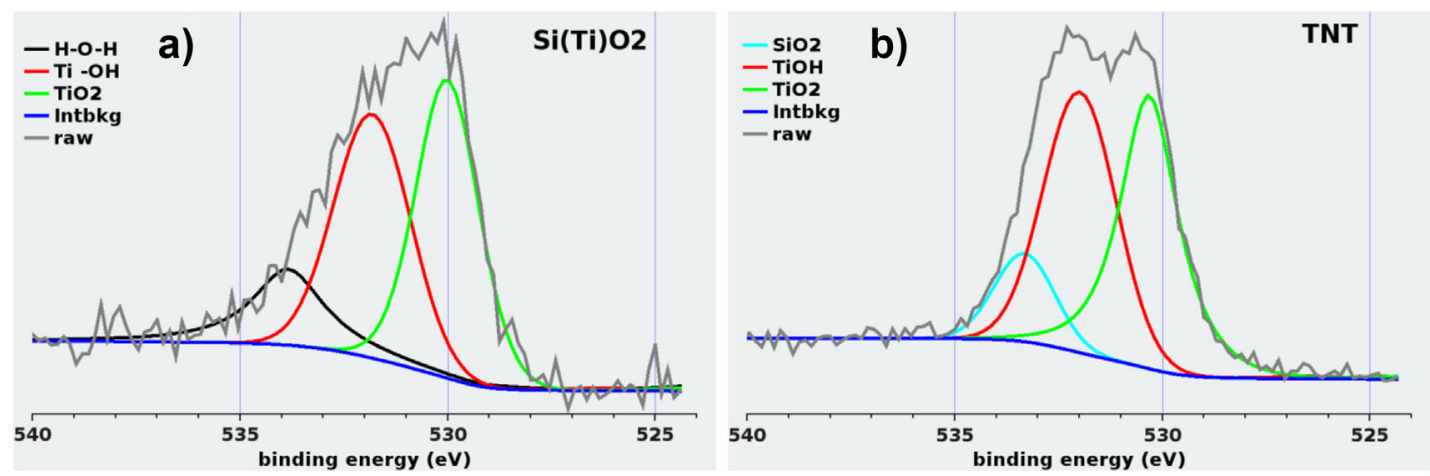

Fig. 9. Decomposition of O 1s line of XPS spectra for the bare waveguide sensor surface (a) and for the TNT layer (b). The TNT shows slightly more TiOH fraction.

the HEK cells (see Figs. 6 and 8), in full agreement with the results of the washing experiments. The OWLS, however, revealed a $30 \%$ higher adhesion in case of the titanate coating compared to the bare waveguide (control) surface. This is encouraging for coating implants (e.g., bone replacements) that need to became assimilated with body tissues.

\subsection{Chemical states and the origin of adsorption and adhesion enhancement}

X-ray photoelectron spectroscopy [62] was used to determine the titanium and the oxygen chemical states $[37,63]$ of the coated and uncoated waveguides (Fig. 9). All titanium detected, in both the titanate and the $\mathrm{Si}_{0.6} \mathrm{Ti}_{0.4} \mathrm{O}_{2}$ chip, prior to any cleaning pretreatment, belonged to one state, which was identified as $\mathrm{Ti}^{4+}$ (note that any species present at $<2 \%$ abundance would not be detected). The oxygen 1s line was split into two components in the recorded spectra because the oxygen in $\mathrm{TiO}_{2}$ and in $\mathrm{OH}$ has a $1.6 \mathrm{eV}$ difference in binding energy $\left(\mathrm{SiO}_{2}\right.$ is indistinguishable from $\left.\mathrm{TiO}_{2}\right)$. The degree of surface hydroxylation was slightly higher in the titanate compared with the $\mathrm{Si}_{0.6} \mathrm{Ti}_{0.4} \mathrm{O}_{2}$ chip ( $\left.30 \%\right)$. The chemical state of the titanium is, therefore, the same in both substrates; the titanate is slightly more hydroxylated. For the calculation of the proteinsubstrate interaction potential, we may assume that the titanate resembles $\mathrm{TiO}_{2}[64] .^{3}$

Therefore, the increased protein adsorption on the TNT presumably corresponds to the increased area available on the nanostructured surface (Fig. 1). The adhesion of living cells was enhanced by the nanostructured TNT layer, as evidenced by the rapid spreading curves recorded by OWLS and the increased resistance to hydromechanical removal (by washing). The enhancement of cell adhesion is in line with the general observations of cell adhesion being enhanced by rough substrata [65-67]. We can exclude any effects of electrostatic interactions between protein and substratum, because at the ionic strength of the medium we used the electrostatic interactions are negligible in comparison with the Lewis acid/base interactions [64].

\section{Summary and outlook}

In summary, titanate nanotube coatings were fabricated from additive-free sols, using a simple, fast, inexpensive, room temperature spin-coating method. The prepared TNT layers were characterized by AFM, showing that the nanostructured films are densely packed. The thicknesses of the coatings were characterized

${ }^{3}$ Of course, the uncoated waveguide contains a significant fraction of silica as well as the titanate-resembling titania. However, the components of the interfacial interaction potential of silica and titania are themselves rather similar [64]. by SE and OWLS, which revealed that the layers are 7-14 nm thick. The chemical state of the surface was examined by XPS; the titanate strongly resembles the titania of the uncoated substrate, implying that the differences in protein adsorption and cell spreading and adhesion may be ascribed to morphological rather than chemical differences between the uncoated $\mathrm{Si}_{0.6} \mathrm{Ti}_{0.4} \mathrm{O}_{2}$ and titanate surfaces.

The maintenance of light-guiding capability of the TNT-coated waveguide biosensors was demonstrated by OWLS.

Protein adsorption experiments revealed that the surfaceadsorbed mass density of BSA was increased on the TNT coating compared to the uncoated surfaces. Living cells spread and adhered better on the TNT substratum.

The results obtained could be further exploited in various directions. Increasing the specific surface area on surface-sensitive optical chips improves their sensitivity by enhancing their analyte capture capacity. The titanate nanotube films described in this paper could potentially be also used as cell adhesive coatings on medical implants. The simplicity and universality of the room temperature fabrication process makes this type of coating a particularly promising candidate for such applications. Future work may include the detailed investigation of cell adhesion, proliferation and alkaline phosphatase-activity on these novel coatings.

The most competitive aspect of the potential exploitation of our results is the ease of application of the coating: it could simply be painted or sprayed on objects of arbitrary geometry and allowed to dry, without the need for special heat treatment or other postapplication processing.

\section{Acknowledgements}

This work was supported by the Lendület program of the Hungarian Academy of Sciences. Support from OTKA K81842 and KMR_12-1-2012-0225 are also gratefully acknowledged. We thank Dr Inna Székács and Beatrix Peter for their help with the cell culture works.

\section{References}

[1] J.J. Ramsden, Experimental methods for investigating protein adsorption kinetics at surfaces, Q. Rev. Biophys. 27 (1994) 41-105.

[2] J.J. Ramsden, D.M. Allen, D.J. Stephenson, J.R. Alcock, G.N. Peggs, G. Fuller, et al., The design and manufacture of biomedical surfaces, CIRP Ann. - Manuf. Technol. 56 (2) (2007) 687-711

[3] K.S. Soppimath, T.M. Aminabhavi, A.R. Kulkarni, W.E. Rudzinski, Biodegradable polymeric nanoparticles as drug delivery devices, J. Control. Release 70 (2001) $1-20$.

[4] O.M. Koo, I. Rubinstein, H. Onyuksel, Role of nanotechnology in targeted drug delivery and imaging: a concise review, Nanomedicine 1 (2005) 193-212.

[5] S.-W. Song, K. Hidajat, S. Kawi, Functionalized SBA-15 materials as carriers for controlled drug delivery: influence of surface properties on matrix-drug interactions, Langmuir 21 (2005) 9568-9575. 
[6] J. Schwartz, M.J. Avaltroni, M.P. Danahy, B.M. Silverman, E.L. Hanson, J.E Schwarzbauer, et al., Cell attachment and spreading on metal implant materials, Mater. Sci. Eng. C 23 (2003) 395-400.

[7] G.W.M. Vandermeulen, H.-A. Klok, Peptide/protein hybrid materials: enhanced control of structure and improved performance through conjugation of biological and synthetic polymers, Macromol. Biosci. 4 (2004) 383-398.

[8] J.J. Ramsden, G.I. Bachmanova, A.I. Archakov, Immobilization of proteins to lipid bilayers, Biosens. Bioelectron. 11 (1996) 523-528.

[9] E. Brynda, M. Houska, J. Skvor, J.J. Ramsden, Immobilisation of multilayer bioreceptor assemblies on solid substrates, Biosens. Bioelectron. 13 (1998) 165-172.

[10] A. Székács, N. Adányi, I. Székács, K. Majer-Baranyi, I. Szendro, Optical waveguide light-mode spectroscopy immunosensors for environmental monitoring, Appl. Opt. 48 (2009) B151-B158.

[11] N. Adányi, I.A. Levkovets, S. Rodriguez-Gil, A. Ronald, M. Váradi, I. Szendro, Development of immunosensor based on OWLS technique for determining Aflatoxin B1 and Ochratoxin A, Biosens. Bioelectron. 22 (2007) 797-802.

[12] P. Roach, D. Farrar, C.C. Perry, Surface tailoring for controlled protein adsorption: effect of topography at the nanometer scale and chemistry, J. Am. Chem. Soc. 128 (2006) 3939-3945

[13] J.J. Ramsden, Biomedical Surfaces, Artech House, Norwood, 2008

[14] H. Frey, R. Haag, Dendritic polyglycerol: a new versatile biocompatiblematerial, J. Biotechnol. 90 (2002) 257-267.

[15] A. Pizzoferrato, A. Vespucci, G. Ciapetti, S. Stea, Biocompatibility testing of prosthetic implant materials by cell cultures, Biomaterials 6 (1985) 346-351.

[16] N. Orgovan, R. Salánki, N. Sándor, Z. Bajtay, A. Erdei, B. Szabó, et al., In-situ and label-free optical monitoring of the adhesion and spreading of primary monocytes isolated from human blood: dependence on serum concentration levels, Biosens. Bioelectron. 54 (2014) 339-344.

[17] N. Orgovan, B. Peter, S. Bősze, J.J. Ramsden, B. Szabó, R. Horvath, Dependence of cancer cell adhesion kinetics on integrin ligand surface density measured by a high-throughput label-free resonant waveguide grating biosensor, Sci. Rep. 4 (2014) 4034.

[18] L. Bacakova, E. Filova, M. Parizek, T. Ruml, V. Svorcik, Modulation of cell adhesion, proliferation and differentiation on materials designed for body implants Biotechnol. Adv. 29 (2011) 739-767.

[19] C.S. Chen, Geometric control of cell life and death, Science 276 (1997) $1425-1428$

[20] R.G. Chapman, E. Ostuni, M.N. Liang, G. Meluleni, E. Kim, L. Yan, et al., Polymeric thin films that resist the adsorption of proteins and the adhesion of bacteria, Langmuir 17 (2001) 1225-1233.

[21] I. Banerjee, R.C. Pangule, R.S. Kane, Antifouling coatings: recent developments in the design of surfaces that prevent fouling by proteins, bacteria, and marine organisms, Adv. Mater. 23 (2011) 690-718.

[22] G. Gyulai, C.B. Penzes, M. Mohai, T. Lohner, P. Petrik, S. Kurunczi, et al., Interfacial properties of hydrophilized poly(lactic-co-glycolic acid) layers with various thicknesses, J. Colloid Interface Sci. 362 (2011) 600-606.

[23] T. McPherson, A. Kidane, I. Szleifer, K. Park, Prevention of protein adsorption by tethered poly (ethylene oxide) layers: experiments and single-chain meanfield analysis, Langmuir 7463 (1998) 176-186.

[24] N. Huang, R. Michel, J. Voros, M. Textor, Poly(L-lysine)-g-poly (ethylene gly$\mathrm{col}$ ) layers on metal oxide surfaces: surface-analytical characterization and resistance to serum and fibrinogen adsorption, Langmuir (2001) 489-498.

[25] H. Garg, G. Bedi, A. Garg, Implant surface modifications: a review, J. Clin. Diagn. Res. 6 (2012) 319-324.

[26] G. Rong, A. Najmaie, J.E. Sipe, S.M. Weiss, Nanoscale porous silicon waveguide for label-free DNA sensing, Biosens. Bioelectron. 23 (2008) 1572-1576.

[27] J.L. Lawrie, Y. Jiao, S.M. Weiss, Size-dependent infiltration and optical detection of nucleic acids in nanoscale pores, IEEE Trans. Nanotechnol. 9 (2010) 596-602.

[28] E. Guillermain, V. Lysenko, R. Orobtchouk, T. Benyattou, S. Roux, A. Pillonnet, et al., Bragg surface wave device based on porous silicon and its application for sensing, Appl. Phys. Lett. 90 (2007) 241116.

[29] Y. Xia, J.A. Rogers, K.E. Paul, G.M. Whitesides, Unconventional methods for fabricating and patterning nanostructures, Chem. Rev. 99 (1999) 1823-1848.

[30] N.L. Rosi, C.A. Mirkin, Nanostructures in biodiagnostics, Chem. Rev. 105 (2005) $1547-1562$

[31] A.S. Aricò, P. Bruce, B. Scrosati, J.-M. Tarascon, W. van Schalkwijk, Nanostructured materials for advanced energy conversion and storage devices, Nat. Mater. 4 (2005) 366-377.

[32] G.A. Hughes, Nanostructure-mediated drug delivery, Dis. Mon. 51 (2005) 342-361.

[33] O. Ai, D. Yang, Y. Li, J. Shi, X. Wang, Z. Jiang, Highly efficient covalent immobilization of catalase on titanate nanotubes, Biochem. Eng. J. 83 (2014) 8-15.

[34] J.H. Forstater, A. Kleinhammes, Y. Wu, Self-assembly of protein-based biomaterials initiated by titania nanotubes, Langmuir 29 (2013) 15013-15021.

[35] N. Swami, Z. Cui, L.S. Nair, Titania nanotubes: novel nanostructures for improved osseointegration, J. Heat Transfer 133 (2011) 034002

[36] A.W. Tan, B. Pingguan-Murphy, R. Ahmad, S.A. Akbar, Review of titania nanotubes: fabrication and cellular response, Ceram. Int. 38 (2012) 4421-4435.

[37] L. Korosi, S. Papp, V. Hornok, A. Oszko, P. Petrik, D. Patko, et al., Titanate nanotube thin films with enhanced thermal stability and high-transparency prepared from additive-free sols, J. Solid State Chem. 192 (2012) 342-350.
[38] S. Mozia, E. Borowiak-Paleń, J. Przepiórski, B. Grzmil, T. Tsumura, M. Toyoda, et al., Physico-chemical properties and possible photocatalytic applications of titanate nanotubes synthesized via hydrothermal method, J. Phys. Chem. Solids 71 (2010) 263-272

[39] C. von Wilmowsky, S. Bauer, In vivo evaluation of anodic $\mathrm{TiO}_{2}$ nanotubes: an experimental study in the pig, J. Biomed. Mater. Res. 89 (2009) 165-171.

[40] S. Beke, L. Korosi, A. Scarpellini, F. Anjum, F. Brandi, Titanate nanotube coatings on biodegradable photopolymer scaffolds, Mater. Sci. Eng. C: Mater. Biol. Appl. 33 (2013) 2460-2463.

[41] N. Kovacs, D. Patko, N. Orgovan, S. Kurunczi, J.J. Ramsden, F. Vonderviszt, et al., Optical anisotropy of flagellin layers: in situ and label-free measurement of adsorbed protein orientation using OWLS, Anal. Chem. 85 (2013) 5382-5389.

[42] D. Patko, B. Gyorgy, A. Nemeth, K.E. Szabó-Taylor, A. Kittel, E.I. Buzas, et al. Label-free optical monitoring of surface adhesion of extracellular vesicles by grating coupled interferometry, Sens. Actuators B: Chem. 188 (2013) 697-701.

[43] R. Horvath, H.C. Pedersen, N. Skivesen, D. Selmeczi, N.B. Larsen, Optical waveguide sensor for on-line monitoring of bacteria, Opt. Lett. 28 (2003)1233-1235

[44] L. Marcotte, M. Tabrizian, Sensing surfaces: challenges in studying the cell adhesion process and the cell adhesion forces on biomaterials, IRBM 29 (2008) 77-88.

[45] T.S. Hug, J.E. Prenosil, M. Morbidelli, Optical waveguide lightmode spectroscopy as a new method to study adhesion of anchorage-dependent cells as an indicator of metabolic state, Biosens. Bioelectron. 16 (2001) 865-874

[46] T.S. Hug, J.E. Prenosil, P. Maier, M. Morbidelli, Optical waveguide lightmode spectroscopy (OWLS) to monitor cell proliferation quantitatively, Biotechnol. Bioeng. 80 (2002) 213-221

[47] R. Horvath, G. Fricsovszky, E. Papp, Application of the optical waveguide lightmode spectroscopy to monitor lipid bilayer phase transition, Biosens. Bioelectron. 18 (2003) 415-428.

[48] R. Kurrat, M. Textor, J. Ramsden, Instrumental improvements in optical waveguide light mode spectroscopy for the study of biomolecule adsorption, Rev. Sci. Instrum. 68 (1997) 2172-2176.

[49] G. Binnig, C. Quate, C. Gerber, Atomic force microscope, Phys. Rev. Lett. 56 (1986) 930-934

[50] R.W. Carpick, M. Salmeron, Scratching the surface: fundamental investigations of tribology with atomic force microscopy, Chem. Rev. 97 (1997) 1163-1194.

[51] F. Giessibl, Advances in atomic force microscopy, Rev. Mod. Phys. 75 (2003) 949-983.

[52] E.S. Gadelmawla, M.M. Koura, T.M.A. Maksoud, I.M. Elewa, H.H. Soliman, Roughness parameters, J. Mater. Process. Technol. 123 (2002) 133-145.

[53] J. Woollam, P. Snyder, M. Rost, Variable angle spectroscopic ellipsometry: a non-destructive characterization technique for ultrathin and multilayer materials, Thin Solid Films 166 (1988) 317-323.

[54] G.J. Jellison, Data analysis for spectroscopic ellipsometry, Thin Solid Films 234 (1993) 416-422.

[55] G.J. Jellison, Spectroscopic ellipsometry data analysis: measured versus calculated quantities, Thin Solid Films 313-314 (1998) 33-39.

[56] P. Langhoff, M. Karplus, Pade summation of the cauchy dispersion equation, J. Opt. Soc. Am. 59 (1969) 863-871.

[57] F. Jenkins, H. White, Fundamentals of Optics, 3rd ed., McGraw-Hill, New York, 1957

[58] K. Tiefenthaler, W. Lukosz, Sensitivity of grating couplers as integrated-optica chemical sensors, J. Opt. Soc. Am. B 6 (1989) 209-220.

[59] R. Kurrat, J. Prenosil, J.J. Ramsden, Kinetics of human and bovine serum albumin adsorption at silica-titania surfaces, J. Colloid Interface Sci. 185 (1997) 1-8.

[60] N. Orgovan, D. Patko, C. Hos, S. Kurunczi, B. Szabó, J.J. Ramsden, et al., Sample handling in surface sensitive chemical and biological sensing: a practical review of basic fluidics and analyte transport, Adv. Colloid Interface Sci. (2014), http://dx.doi.org/10.1016/j.cis.2014.03.011 (in press).

[61] J.J. Ramsden, R. Horvath, Optical biosensors for cell adhesion, J. Recept. Signal Transduct. Res. 29 (2009) 211-223.

[62] C.S. Fadley, X-ray photoelectron spectroscopy: progress and perspectives, J Electron Spectrosc. Relat. Phenom. 178-179 (2010) 2-32.

[63] J.J. Ramsden, R. Tóth-Boconádi, Pulsed photoelectrochemistry of titanium dioxide, J. Chem. Soc. Faraday Trans. 86 (1990) 1527-1533.

[64] M.G. Cacace, E.M. Landau, J.J. Ramsden, The Hofmeister series: salt and solvent effects on interfacial phenomena, Q. Rev. Biophys. 30 (1997) 241-277.

[65] T.-W. Chung, D.-Z. Liu, S.-Y. Wang, S.-S. Wang, Enhancement of the growth of human endothelial cells by surface roughness at nanometer scale, Biomaterials 24 (2003) 4655-4661.

[66] M. Lampin, Warocquier-Clérout, C. Legris, M. Degrange, M.F. Sigot-Luizard, Correlation between substratum roughness and wettability, cell adhesion, and cell migration, J. Biomed. Mater. Res. 36 (1997) 99-108.

[67] R.L. Price, K. Ellison, K.M. Haberstroh, T.J. Webster, Nanometer surface roughness increases select osteoblast adhesion on carbon nanofiber compacts, J. Biomed. Mater. Res. A 70 (2004) 129-138

[68] A. Aref, R. Horvath, J.J. Ramsden, Spreading kinetics for quantifying cell state during stem cell differentiation, J. Biol. Phys. Chem. $10(2010)$ $145-151$

[69] J. Vörös, J.J. Ramsden, G. Csucs, I. Szendrő, S.M. De Paul, M. Textor, et al., Optica grating coupler biosensors, Biomaterials 23 (2002) 3699-3710. 